Check for updates

Cite this: RSC Adv., 2017, 7, 39471

\title{
Facile fabrication of raspberry-like composite microspheres for the construction of superhydrophobic films and applications in highly efficient oil-water separation $\dagger$
}

\begin{abstract}
Mingguang Yu, (D) a Qing Wang, ${ }^{\text {tb }}$ Min Zhang, ${ }^{a}$ Qianjun Deng ${ }^{a}$ and Dongchu Chen ${ }^{a}$
Inspired by the "lotus effect", we proposed a facile synthetic route toward raspberry-like $\mathrm{PS}_{\mathrm{SSiO}}$ microspheres, which further lead to superhydrophobic surfaces. In this approach, monodispersed polystyrene (PS) microspheres were first synthesized via dispersion polymerization using polyvinylpyrrolidone (PVP) as stabilizer. The obtained PS microspheres were then used as template microspheres for biomimetic silification using tetraethyl orthosilicate (TEOS) as precursor. Upon adjusting the molecular weight of PVP and the concentration of $\mathrm{NH}_{3} \cdot \mathrm{H}_{2} \mathrm{O}$, the surface roughness of $\mathrm{PSASiO}_{2}$ microspheres can be well controlled. Furthermore, after hydrophobization treatment, by dropcasting the raspberry-like $\mathrm{PS} \mathrm{SSiO}_{2}$ microspheres onto a glass slide, dual-scale films were obtained, which had a similar surface morphology to that of the lotus leaf, exhibiting a water contact angle of $163.3^{\circ}$ and water contact angle hysteresis of $4^{\circ}$. In addition, the oil-water separation ability of hydrophobic raspberry microsphere treated steel mesh was investigated. The results demonstrated excellent oil-water separation efficiency and reusability. This facile and robust synthesis technique for constructing a superhydrophobic surface hold great potential application in versatile and large-scale oil-

water separation.
\end{abstract}

Received 30th June 2017

Accepted 7th August 2017

DOI: $10.1039 / c 7 r a 07250 c$

rsc.li/rsc-advances

\section{Introduction}

Materials with superwetting ability have attracted wide attention from both academia and industry due to their great potential applications in self-cleaning, ${ }^{1-7}$ combatting bacteria, ${ }^{\mathbf{8}, 9}$ agriculture, ${ }^{10}$ separation of liquids, ${ }^{11-19}$ printing and reprography, ${ }^{20,21}$ etc. In many scientific studies, the "lotus effect" has been reported as a non-wetting phenomenon, which is governed by a specific nano-micro structured surface topography and low surface energy. Therefore, a superhydrophobic surface can be obtained by lowering the surface energy and adjusting the surface roughness. ${ }^{20}$

Inspired by superhydrophobic biomaterials, many synthesis strategies have been established to mimic the dual-scale rough hierarchical morphology for the fabrication of superhydrophobic surfaces, ${ }^{22-32}$ such as plasma polymerization/ etching, ${ }^{33,34}$ chemical vapor deposition, ${ }^{31}$ electro spinning, ${ }^{35,36}$ etc. However, the high cost materials, relatively complicated

${ }^{a}$ School of Materials Science and Energy Engineering, Foshan University, Foshan 528000, China

${ }^{b}$ State Key Laboratory of Pulp \& Paper Engineering, South China University of Technology, Guangzhou 510640, China. E-mail: wangqing_1216@126.com

$\dagger$ Electronic supplementary information (ESI) available. See DOI: $10.1039 / \mathrm{c} 7 \mathrm{ra} 07250 \mathrm{c}$ production equipment or operation procedures largely affect the actual application of the superhydrophobic materials.

Recently, raspberry-like colloidal microspheres with dualsized hierarchical structures have gained increasing attention for their potential applications in the construction of superhydrophobic films. ${ }^{36-43}$ In most cases, inorganic/organic raspberry-like microspheres were used in assemble superhydrophobic coatings. ${ }^{44-48}$ A variety of methods have been developed for the successful fabrication of these samples. ${ }^{47,49,50}$ The silica-coated or titania-coated raspberry-like microspheres was usually obtained by acid-base interaction, electrostatic interaction between the silanol groups and the functional groups on templates. Zhang et al. ${ }^{51}$ reported a method for the preparation of raspberry microspheres using non-covalent interactions between organic and inorganic components. They first introduced the functional group of acrylic acid (AA) in the preparation of polystyrene (PS) microspheres to prepare polyacrylic acid functionalized PS microspheres. Due to the strong hydrogen bonding between the carboxyl groups in the polyacrylic acid and the siloxanes possessed by the ethyl orthosilicate (TEOS), TEOS is adsorbed to the PS microspheres surface, and then in situ hydrolysis into nano-silica on the surface of PS

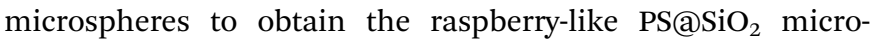
spheres. Caruso et al..$^{52}$ reported the method of lays of selfassembly (LBL) to prepare $\mathrm{PS@SiO}{ }_{2}$ raspberry-like structural 
microspheres utilized non-covalent interactions of the negatively charged $\mathrm{SiO}_{2}$ microspheres and positively charged polydimethylenediammonium chloride (PDDA) modified PS microspheres. Raspberry-like microspheres can also obtained via surface modification/polymerization method, in which silica microspheres were first surface treated with methacryloxypropyltrimethoxysilane then copolymerized with vinyl monomers. ${ }^{53}$ However, many of these methods used toxic or high cost materials, wild reaction conditions, or multistep processes. ${ }^{33}$

Poly(vinylpyrrolidone) (PVP) was an amphiphilic, hypotoxicity, nonionic polymer which was widely used in scientific research and industry. In dispersion polymerization system, PVP was a very commonly used stable dispersant. After nucleation, it can be adsorbed or grafted on the surface to stabilize the nucleus of the polymer microspheres. ${ }^{54,55}$ Moreover, the tertiary amine groups presented in the molecular structure of PVP had excellent coordination and adsorption ability to adsorb a broad range of different materials such as metals, metal oxides and inorganic source precursors. They can be adsorbed on the surface of microspheres and in situ induced or hydrolysed into nanoparticles to obtain organic and inorganic hybrid materials.

Herein, we present a general, simple and fast method to fabricate raspberry-like $\mathrm{PS@SiO}{ }_{2}$ composite microspheres, which was based on the use of PVP. In detail, monodispersed PS microspheres were first synthesized via dispersion polymerization using PVP as stabilizer. Then the PS microspheres were used as templates for the biomimetic silification using tetraethyl orthosilicate (TEOS) as precursor. During the process, PVP on the one hand stabilized styrene monomer forming monodispersed PS microspheres, on the other hand worked as the linkers to bridge PS microspheres and TEOS to induce the formation of the raspberry-like $\mathrm{PS@SiO}{ }_{2}$ composite microspheres. In addition, surface roughness of the as-prepared raspberry-like composite microspheres could be well controlled upon adjusting the molar mass of PVP and the concentration of ammonia. Furthermore, steel mesh treated with hydrophobized raspberry-like $\mathrm{PS} @ \mathrm{SiO}_{2}$ microspheres showed excellent oil-water separation efficiency and reusability. This facile synthesis technique for construction the superhydrophobic surface hold great potential application in versatile and large-scale oil-water separation.

\section{Experimental}

\section{Materials}

Styrene (St, 99.9\%, Aldrich) was freshly distilled under vacuum and stored under freeze prior to use. PVP with an average molecular weight of $10 \mathrm{~kg} \mathrm{~mol}^{-1}, 40 \mathrm{~kg} \mathrm{~mol}^{-1}, 58 \mathrm{~kg} \mathrm{~mol}^{-1}$ and $130 \mathrm{~kg} \mathrm{~mol}{ }^{-1}$ were purchased from Aldrich and used as received. Azobisisobutyronitrile (AIBN, Aldrich, 99\%) was purified by recrystallization and stored in a refrigerator prior to use. Hexadecyltrimethoxysilane (HDTMS, >99\%) were purchased from Aldrich and used as received. Tetraethyl orthosilicate (TEOS, >99\%), anhydrous ethanol, and aqueous ammonia solution $\left(\mathrm{NH}_{3} \cdot \mathrm{H}_{2} \mathrm{O}, 25 \mathrm{wt} \%\right)$ were purchased from Guangzhou Chemical Reagent Co. and used as received. Polyurethane (PU) sponges and steel mesh $(\Phi=30 \mathrm{~mm}, 200$ mesh sieve) were purchased from a local store, which were cleaned by ethanol and deionized water, respectively.

\section{Preparation of monodispersed PS microspheres}

Monodispersed polystyrene (PS) microspheres were performed in ethanol-water mixture $(80 / 20, \mathrm{w} / \mathrm{w})$ at $70{ }^{\circ} \mathrm{C}$ via dispersion polymerization. In a typical procedure, styrene $(2.0 \mathrm{~g}, 10 \mathrm{wt} \%$ relative to the reaction system), stabilizer PVP (0.3 g, $15 \mathrm{wt} \%$ relative to monomer) and AIBN $(0.04 \mathrm{~g}, 2 \mathrm{wt} \%$ relative to monomer) were dissolved in $\mathrm{EtOH} / \mathrm{H}_{2} \mathrm{O}$ mixture $(18.0 \mathrm{~g}$, $80 / 20, w / w)$. The mixture was purged with nitrogen for 30 minutes under stirring, sealed, and then conducted at $70{ }^{\circ} \mathrm{C}$ for 8 hours.

The reaction mixture was separated by centrifugation, rinsed with ethanol/water mixture and centrifuged repeatedly.

\section{Preparation of raspberry-like $\mathrm{PS} @ \mathrm{SiO}_{2}$ microspheres}

The raspberry-like $\mathrm{PS} @ \mathrm{SiO}_{2}$ microspheres were prepared by a modified Stöber method. ${ }^{51}$ Typically, $100 \mathrm{mg}$ of monodispersed PS microspheres were dispersed in reaction mixture in a $250 \mathrm{~mL}$ flask containing $100 \mathrm{~mL}$ ethanol and $20 \mathrm{~mL}$ deionized water and a certain amount of ammonium hydroxide (25 wt\%) under gentle magnetic stirring at room temperature. Then $0.2 \mathrm{~mL}$ of TEOS was slowly added to the solution. The mixture was stirred vigorously for $24 \mathrm{~h}$ at room temperature. After the hydrolysis reaction of TEOS, the mixture was centrifuged and rinsed with ethanol/water mixture and centrifuged

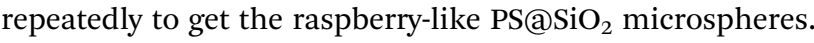

\section{Fabrication of the superhydrophobic surfaces}

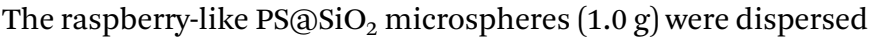
into $20 \mathrm{~mL}$ ethanol solution containing HDTMS (1 wt\%) and the solution was magnetic stirring for 2 hours. Then a certain amount of sample emulsion was carefully drop-cast onto glass, paper, cotton fibres, polyurethane (PU) sponge and steel mesh in order to generate the dual-scale films, and then these films were heated for $5 \mathrm{~min}$ at $60{ }^{\circ} \mathrm{C}$ in an oven to form superhydrophobic surfaces.

\section{Characterization}

FT-IR spectra were recorded on a Spectrum 2000 Perkin Elmer FT-IR spectrometer. X-Ray photoelectron spectroscopy (XPS) spectra were recorded on a Thermo Electron Corporation Escalab 250 spectrometer with $\mathrm{Li} \mathrm{K} \alpha$ radiation $(20 \mathrm{eV})$ as the exciting source. All the samples were ground into powder and dried under infrared lamp before examination. TEM measurements were carried out on FEI Tecnai $G^{2}$ Spirit transmission electron microscopy. Samples were diluted with ethanol and ultrasonicated for $10 \mathrm{~min}$ to make the suspensions with a concentration of $0.1 \mathrm{wt} \%$ and then dried onto carbon-coated copper grids before examination. SEM measurements were carried out on a Termal field-emission scanning electron microscope (FESEM, Quanta 400F) at $15 \mathrm{kV}$. Samples were dispersed into ethanol with a concentration of $0.1 \mathrm{wt} \%$ via 
ultrasonication for $10 \mathrm{~min}$. The dispersions were dip-casting onto the surface of glass and coated with a thin layer of gold before examination. Thermogravimetric analysis (TGA) was performed on a Q600 instrument (TA Instruments, New Castle, USA). All samples were heated from $40{ }^{\circ} \mathrm{C}$ to $600{ }^{\circ} \mathrm{C}$ at a heating rate of $20{ }^{\circ} \mathrm{C} \mathrm{min}^{-1}$. Contact angles (CAs) were measured on a Dataphysics OCA20 Contract-Angle System with liquid droplets of $5 \mu \mathrm{L}$. And the reported values represented the averages of at least 5 different positions' measurements. The separation efficiency was calculated according to $\theta=\left(m_{1} / m_{0}\right) \times 100 \%$, where $m_{0}$ and $m_{1}$ are the mass of the residual liquid before and after the separation process, respectively. ${ }^{\mathbf{1 7}}$

\section{Results and discussion}

\section{Composition characterization of monodispersed PS microspheres and raspberry-like $\mathrm{PS} @ \mathrm{SiO}_{2}$ composite microspheres}

As is well known, TEOS can be used to prepare a variety of nanostructured silicon nanoparticles. Polymers containing secondary amines and tertiary amines could catalyse the hydrolysis and condensation polymerization of TEOS, which may strongly adsorbed TEOS by the charge effect of the amine sites. Here, the monodispersed PS microspheres were prepared via one-pot dispersion polymerization using PVP as stabilizer (Scheme 1). The presence of PVP ensured strong static adsorption of the PS microspheres with the TEOS, which arose from the presence of a highly polar amide group within the pyrrolidone ring of PVP. ${ }^{52}$ Under alkaline condition $\left(\mathrm{NH}_{3} \cdot \mathrm{H}_{2} \mathrm{O}\right.$ as catalyst), TEOS was in situ hydrolysed into silica nanoparticles on the surface of the PS microspheres.

FT-IR spectra was used to record the structure of the PS and raspberry-like PS@SiO ${ }_{2}$ composite microspheres. As shown in Fig. 1a, absorption peaks at 3025, 1600, 1492, 1450, 757, 697 $\mathrm{cm}^{-1}$ corresponded to the characteristic absorption peaks of phenyl group, the peaks at 2920 and $2848 \mathrm{~cm}^{-1}$ can be attributed to the methylene and methenyl groups of polystyrene main chain. The peak at $1682 \mathrm{~cm}^{-1}$ and the peak at $1291 \mathrm{~cm}^{-1}$ attributed to the $\mathrm{C}=\mathrm{O}$ stretching vibration and the $\mathrm{C}-\mathrm{N}$ stretching vibration of the pyrrolidone ring in PVP, which means PVP was located at the surface of PS spheres. After coated with $\mathrm{SiO}_{2}$, there were several new absorbance signals appearing in FT-IR spectra. As shown in Fig. 1b, $463 \mathrm{~cm}^{-1}$ and $753 \mathrm{~cm}^{-1}$ were the antisymmetric stretching vibration peak and symmetric stretching vibration peak of Si-O respectively, and the Si-O-Si stretching vibration band was split into two peaks (1153 and $1093 \mathrm{~cm}^{-1}$ ). The absorption peak of Si-OH was at

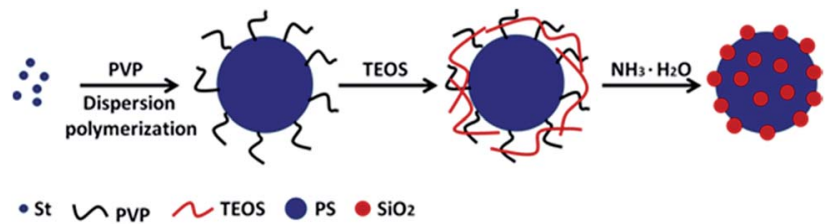

Scheme 1 Schematic illustration of procedure for preparation of raspberry-like $\mathrm{PS} \mathrm{aSiO}_{2}$ composite microspheres.

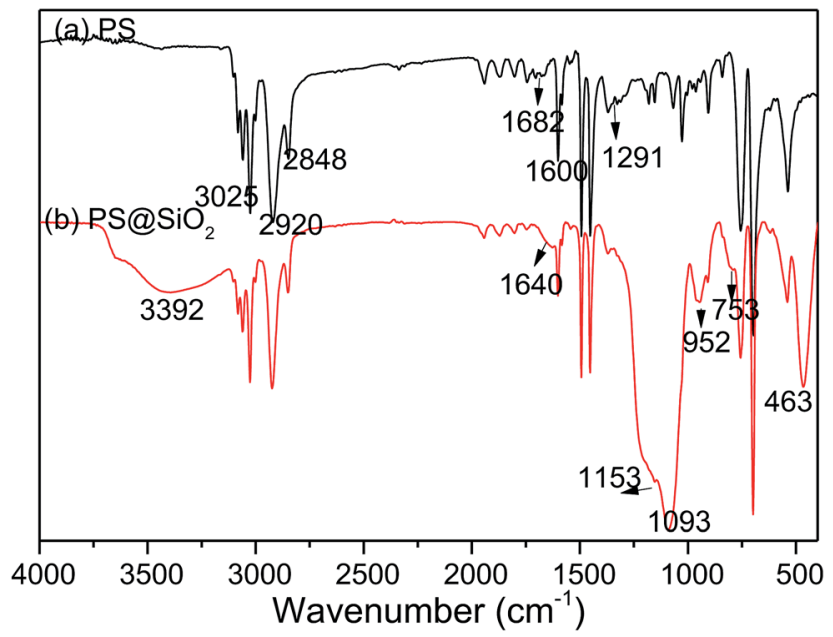

Fig. 1 FT-IR spectra of PS and $\mathrm{PS} \mathrm{SSiO}_{2}$ composite microspheres.

$952 \mathrm{~cm}^{-1}$. The peaks at $1640 \mathrm{~cm}^{-1}$ and $3392 \mathrm{~cm}^{-1}$ were the bending vibration peak of $\mathrm{H}-\mathrm{O}-\mathrm{H}$ and the antisymmetric stretching vibration peak of $\mathrm{O}-\mathrm{H}$. These features indicated that $\mathrm{SiO}_{2}$ were successfully coated to the PS microspheres.

XPS was further employed to determine the surface composition of PS and raspberry-like $\mathrm{PS@SiO}{ }_{2}$ composite microspheres, shown in Fig. 2. The typical information depth of XPS was $5 \mathrm{~nm}$. The spectra of PS microspheres revealed the presence of carbon (284.8 eV) and oxygen (532.82 eV). The peak of oxygen was corresponding to the stabilizer PVP, which proved PVP was adsorption onto the surface of PS microspheres during dispersion polymerization. In the XPS spectra of the raspberry-like PS@SiO ${ }_{2}$ composite microspheres, the relative atomic concentration of oxygen increased from $3.62 \%$ to $10.07 \%$, together with the appearance of two typical peaks with binding energies of $155.24 \mathrm{eV}$ ( $\mathrm{Si} 2 \mathrm{~s}$ ) and $103.58 \mathrm{eV}$ (Si 2p), strongly confirmed the presence of silica nanoparticles on the surface of the PS microspheres.

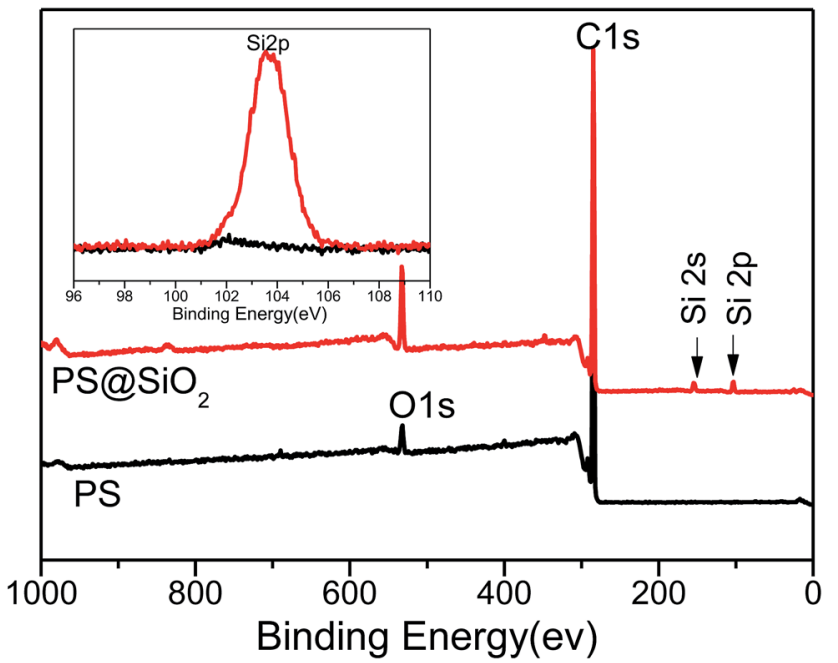

Fig. 2 XPS full-scan spectra of the PS microspheres and $\mathrm{SiO}_{2}$ coated PS microspheres. 


\section{Effect of the concentrations of $\mathrm{NH}_{3} \cdot \mathrm{H}_{2} \mathrm{O}$ on raspberry-like} ${\mathrm{PS} @ S i O_{2}}_{2}$ composite microspheres

According to the classic Stöber process, the surface morphologies and roughness of the silica coated PS microspheres could be easily tuned by varying the reaction conditions, such as the catalyst, the temperature, medium, and time. As is well known, ammonia was often used as the catalyst causing the formation of spherical silica nanoparticles. So in this study, the influence of the amount of ammonia on the surface roughness of the silica modified PS microspheres was studied. The nano- $\mathrm{SiO}_{2}$ diameter and coverage degree on the surface of PS microspheres could be tailored simply by varying the concentration of $\mathrm{NH}_{3} \cdot \mathrm{H}_{2} \mathrm{O}$.

Monodispersed PS microspheres synthesized using PVP-40 as stabilizer were first used as templates to fabricate raspberry-like $\mathrm{PS} @ \mathrm{SiO}_{2}$ composite microspheres in the one-step sol-gel process. Fig. 3 showed SEM images of raspberry-like $\mathrm{PS} @ \mathrm{SiO}_{2}$ composite microspheres obtained at varied $\mathrm{NH}_{3} \cdot \mathrm{H}_{2} \mathrm{O}$ concentrations. When the $\mathrm{NH}_{3} \cdot \mathrm{H}_{2} \mathrm{O}$ volume was $0.5 \mathrm{~mL}$, the PS template was densely and uniformly covered by the silica nanoparticles. The PS surface roughness had no intuitionistic improvement. When the $\mathrm{NH}_{3} \cdot \mathrm{H}_{2} \mathrm{O}$ volume increased to $1.0 \mathrm{~mL}$, the roughness of the PS surface became more pronounced and small humps with size distribution of $c a$. $50 \mathrm{~nm}$ were presented on the PS template surface (Fig. 3c). With the content of $\mathrm{NH}_{3} \cdot \mathrm{H}_{2} \mathrm{O}$ increased from 1.0 to $2.0 \mathrm{~mL}$, the
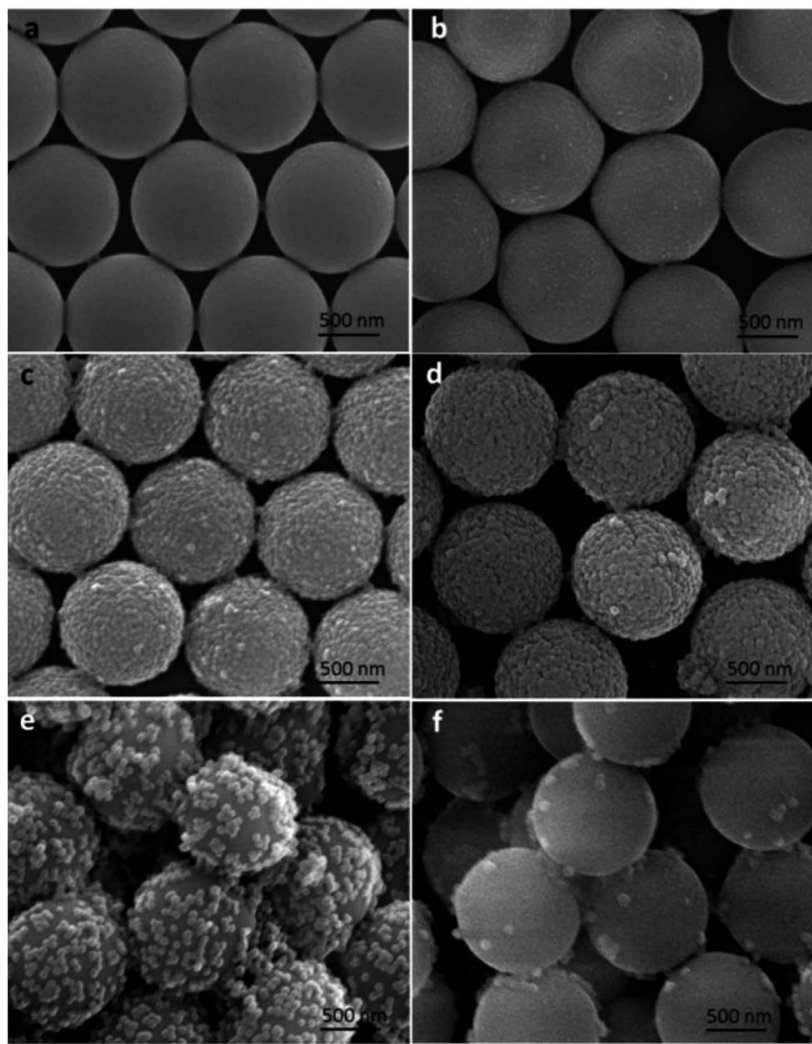

Fig. 3 FESEM images of (a) PS template spheres and (b-e) $\mathrm{PS} \mathrm{aSiO}{ }_{2}$ microspheres prepared with different volumes of $\mathrm{NH}_{3} \cdot \mathrm{H}_{2} \mathrm{O}$ : (b) $0.5 \mathrm{~mL}$; (c) $1.0 \mathrm{~mL}$; (d) $2.0 \mathrm{~mL}$; (e) $3.0 \mathrm{~mL}$ and (f) $4.0 \mathrm{~mL}$. ${\mathrm{PS} @ \mathrm{CSO}_{2}}$ composite microspheres: PVP-40. roughness of the PS surface became more pronounced because of the growth of the nodules. The diameter of $\mathrm{SiO}_{2}$ nanoparticles on the surface of PS microspheres increased to ca. $100 \mathrm{~nm}$ (Fig. 3d). Although the diameter of silica nanoparticles increased with the addition of $\mathrm{NH}_{3} \cdot \mathrm{H}_{2} \mathrm{O}$, further increase the volume of $\mathrm{NH}_{3} \cdot \mathrm{H}_{2} \mathrm{O}$ would decreased the coverage degree of silica on the PS microspheres, such as $3.0 \mathrm{~mL}$ and $4.0 \mathrm{~mL}$ (Fig. $3 \mathrm{e}$ and f). This may due to that with the speeded up of the hydrolysis, it became harder for the PVP adsorbing TEOS onto the surface of PS microspheres.

This raspberry-like structure had also been confirmed by TEM, as shown in Fig. 4. In comparison with PS templates

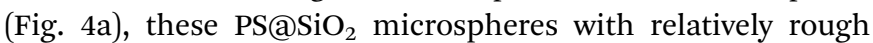
surfaces and slightly larger sizes were observed after the silification. The average diameter measured from the TEM images of $\mathrm{PS} @ \mathrm{SiO}_{2}$ microspheres (Fig. 4b-d) increased from 837 to $928 \mathrm{~nm}$ with the content of $\mathrm{NH}_{3} \cdot \mathrm{H}_{2} \mathrm{O}$ increased from 0.5 to $2.0 \mathrm{~mL}$ during the silification process. But further increase the contents of $\mathrm{NH}_{3} \cdot \mathrm{H}_{2} \mathrm{O}$ would decrease the cover density of silica, which was confirmed by Fig. 4e and f.

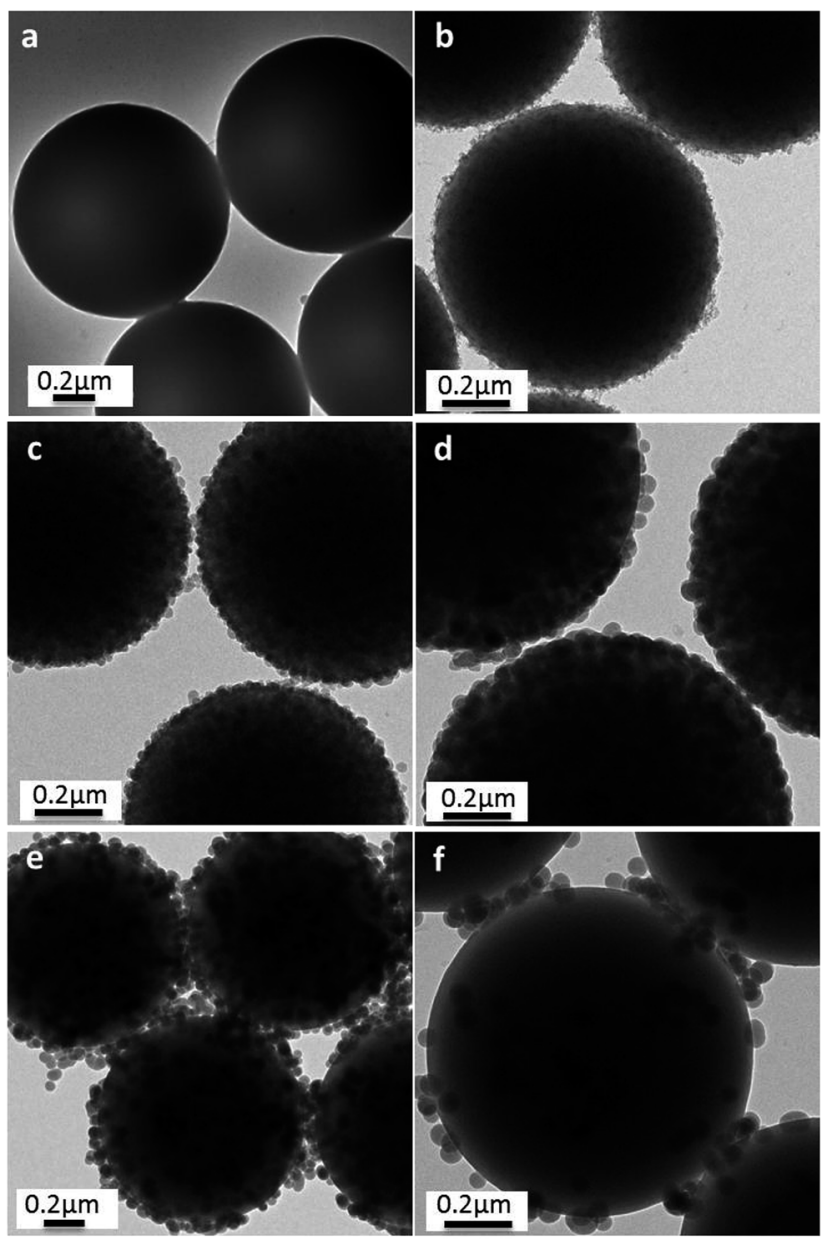

Fig. 4 TEM images of (a) PS template spheres and (b-e) $\mathrm{PSCSiO}_{2}$ microspheres prepared with different volumes of $\mathrm{NH}_{3} \cdot \mathrm{H}_{2} \mathrm{O}$ : (b) $0.5 \mathrm{~mL}$; (c) $1.0 \mathrm{~mL}$; (d) $2.0 \mathrm{~mL}$; (e) $3.0 \mathrm{~mL}$ and (f) $4.0 \mathrm{~mL}$. $\mathrm{PSOSiO}_{2}$ composite microspheres: PVP-40. 
Thermogravimetric analysis was used to quantitate the amount of silica nanoparticles deposited on the PS surface (Fig. 5). The PS template was decomposed completely with residue content of $0.825 \%$ (Fig. 5a). And the residue content slightly increased to $5.21 \%$ after hydrolysis of TEOS with $0.5 \mathrm{~mL}$ $\mathrm{NH}_{3} \cdot \mathrm{H}_{2} \mathrm{O}$ (Fig. 5b), illustrating that the silica nanoparticles were incorporated onto the PS surface. As shown in Fig. 5c-e, with increasingly employing $\mathrm{NH}_{3} \cdot \mathrm{H}_{2} \mathrm{O}$ from $1.0 \mathrm{~mL}, 2.0 \mathrm{~mL}$ to $3.0 \mathrm{~mL}$, the final residue weight of the $\mathrm{PS} @ \mathrm{SiO}_{2}$ were $16.96 \%$, $20.82 \%$ and $24.55 \%$, demonstrating that more silica was incorporated onto PS template. But as the content of $\mathrm{NH}_{3} \cdot \mathrm{H}_{2} \mathrm{O}$ increased to $4.0 \mathrm{~mL}$ (Fig. 5f), the final residue weight of the raspberry like $\mathrm{PS@SiO} \mathrm{Si}_{2}$ decreased to $3.91 \%$. The results of thermogravimetric analysis were consistent with those of SEM and TEM in Fig. 3 and 4. The use of $\mathrm{NH}_{3} \cdot \mathrm{H}_{2} \mathrm{O}$ with $2.0-3.0 \mathrm{~mL}$ appeared to be an optimum for this system, for it provided sufficient surface roughness and silica coverage.

\section{Effect of the molar mass of PVP}

The mass weight of the PVP used played an important role in the stability of the microspheres during the hydrolysis process, since it provided the affinity of tetraethyl orthosilicate with PS template and led TEOS hydrolysis into silica nanoparticles in situ on the surfaces of PS microspheres to obtain raspberry-like PS@SiO ${ }_{2}$ composite microspheres. So the PVP molar mass had an important impact on the surface roughness of raspberry-like microspheres and silica coverage. In this study, the PVP with average molar masses of $10 \mathrm{~kg} \mathrm{~mol}{ }^{-1}$ (PVP-10), $40 \mathrm{~kg} \mathrm{~mol}$ (PVP-40), $58 \mathrm{~kg} \mathrm{~mol}^{-1}$ (PVP-58), and $130 \mathrm{~kg} \mathrm{~mol}^{-1}$ (PVP-130) were not only used as stabilizer to prepared PS microspheres, but also used as an anchor to adsorb and in situ hydrolysis of TEOS into silica nanoparticles on the surface of PS microspheres, and to examine how the molar mass of PVP affected the

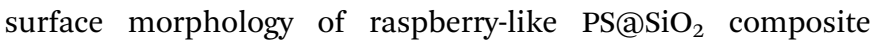
microspheres.

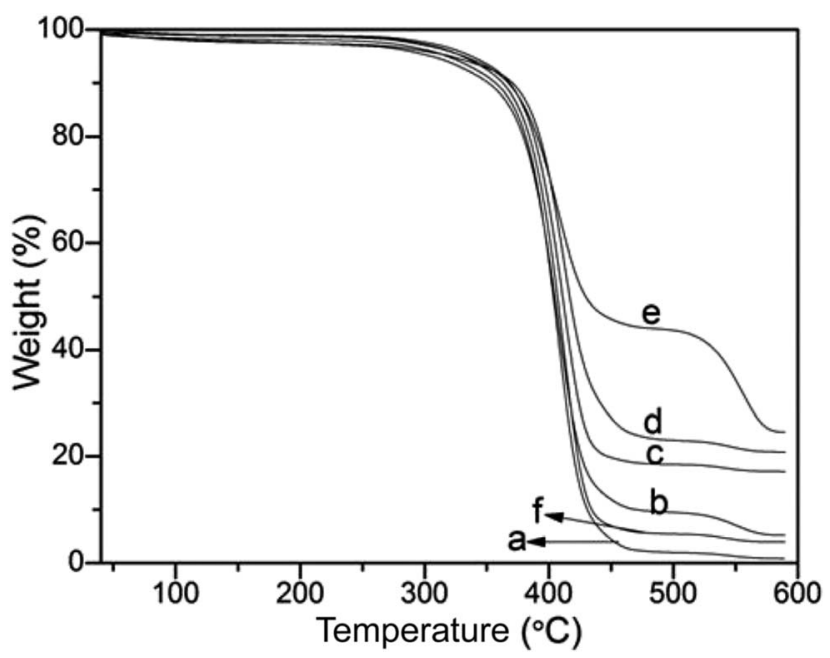

Fig. 5 Thermogravimetric analysis (TGA) curves of (a) PS template microspheres and $(b-e) \mathrm{PS} \mathrm{CSiO}_{2}$ microspheres prepared with different volumes of $\mathrm{NH}_{3} \cdot \mathrm{H}_{2} \mathrm{O}$ : (b) $0.5 \mathrm{~mL}$; (c) $1.0 \mathrm{~mL}$; (d) $2.0 \mathrm{~mL}$; (e) $3.0 \mathrm{~mL}$ and (f) $4.0 \mathrm{~mL}$. PS@SiO 2 composite microspheres: PVP-40.
Fig. 6 showed the SEM images of raspberry-like PS@SiO composite microspheres prepared with different molar mass of PVP as stabilizer. It could be clearly observed that all the four runs yielded the raspberry-like $\mathrm{PS} @ \mathrm{SiO}_{2}$ composite spheres and the $\mathrm{SiO}_{2}$ nanoparticles were evenly distributed on the surface of PS microspheres. Meanwhile, it was found that with the increase of chain length of PVP, the mean diameter of the PS spheres decreased from $2.2 \mu \mathrm{m}$ to less than $1 \mu \mathrm{m}$ and the monodispersity deterioration which was similar with other reported dispersion polymerization system to prepare polymer

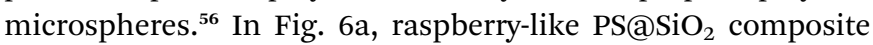
microsphere fabricated with PVP-10 stabilized PS microsphere templates, it had a relatively smooth silica-covered aggregates and homogeneous coating thickness. When the molar mass of PVP increased to PVP-40 and PVP-58, the surface of raspberrylike PS@SiO ${ }_{2}$ composite microsphere turned to more roughness and densest (Fig. 6b and c). The microspheres coated with PVP-130 had a much more inhomogeneous coating thickness and some silica microspheres with multiple layers were observed, but both the PS microspheres and raspberry-like $\mathrm{PS} @ \mathrm{SiO}_{2}$ composite microsphere showed polydispersity, which were shown in Fig. 6d and S1d. $\dagger$

These changes have also been confirmed by TEM, as shown in Fig. 7, in which a close inspection among the raspberry-like $\mathrm{PS} @ \mathrm{SiO}_{2}$ microspheres was observed. The thickness of the silica shell were about $40 \mathrm{~nm}, 57 \mathrm{~nm}, 60 \mathrm{~nm}$ and $100 \mathrm{~nm}$, respectively, with the chain length of PVP increased from $10 \mathrm{~kg} \mathrm{~mol}^{-1}, 40 \mathrm{~kg} \mathrm{~mol}{ }^{-1}, 58 \mathrm{~kg} \mathrm{~mol}^{-1}$ and $130 \mathrm{~kg} \mathrm{~mol}^{-1}$. The results indicated that silica was growing onto the PS microspheres directly. Moreover, longer chain length of PVP can provide more tertiary amine sites which can strongly adsorbed TEOS on the surface of the PS particles and subsequently in situ hydrolysis into silica nanoparticles. In this process, the abundant amount of PVP was not only involving in dispersion polymerization forming monodispersed PS microspheres, but also

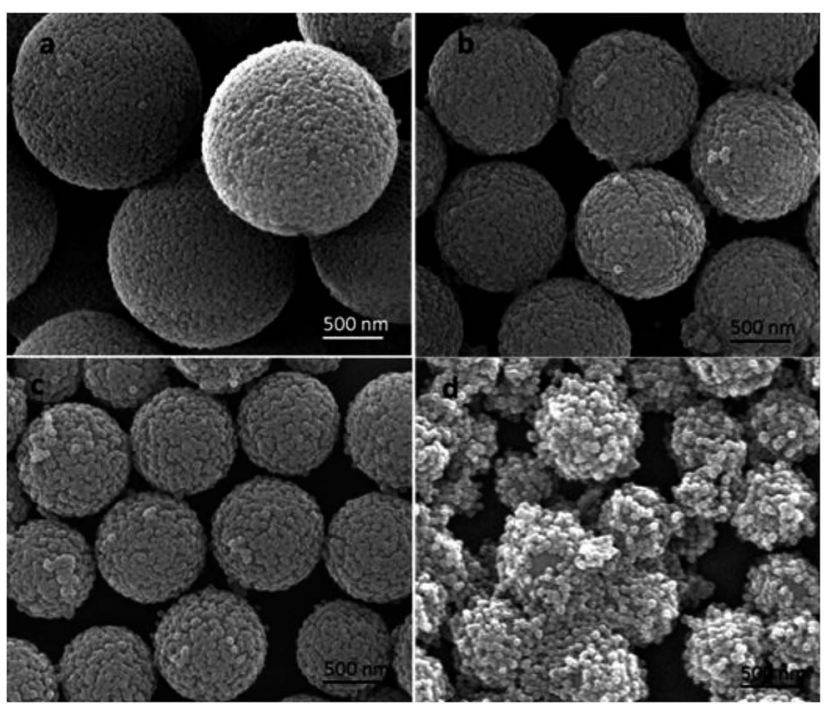

Fig. 6 SEM images of silica coated PS with: (a) PVP-10; (b) PVP-40, (c) PVP-58 and (d) PVP-130. $\mathrm{NH}_{3} \cdot \mathrm{H}_{2} \mathrm{O}=2 \mathrm{~mL}$. 

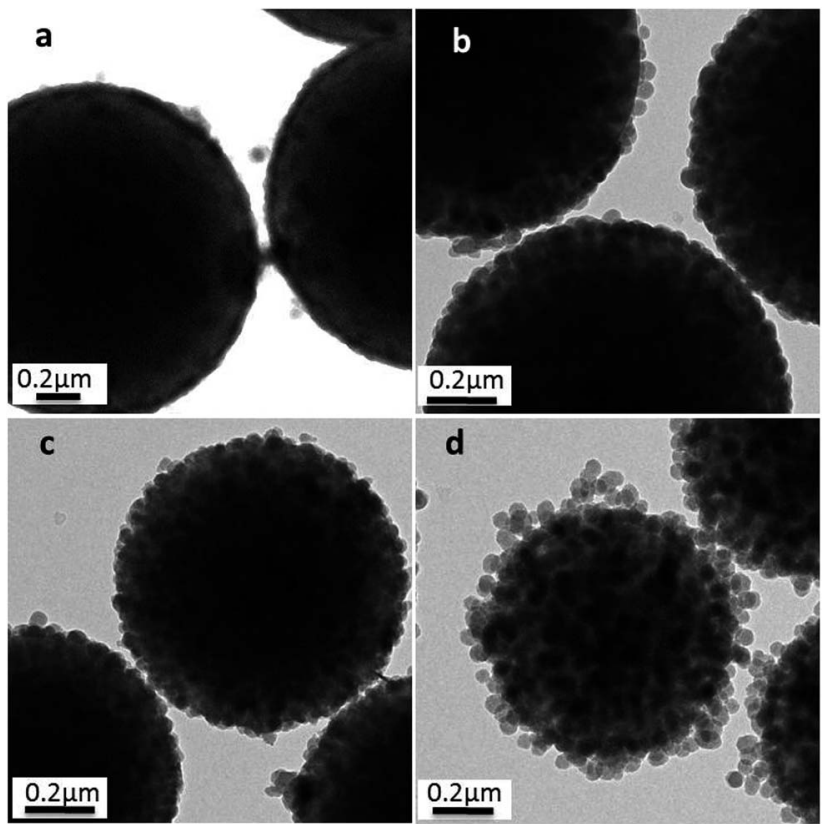

Fig. 7 TEM images of silica coated PS with: (a) PVP-10; (b) PVP-40, (c) PVP-58 and (d) PVP-130. $\mathrm{NH}_{3} \cdot \mathrm{H}_{2} \mathrm{O}=2 \mathrm{~mL}$

playing a pivotal role in the biomimetic silification, since the tertiary amine sites may strongly adsorbed TEOS and catalyze the hydrolysis and condensation polymerization. Both of which are key factors for the formation of raspberry-like ${\mathrm{PS} @ \mathrm{SiO}_{2}}$ composite microspheres.

Thermogravimetric analysis was also used to quantitatively determine the effects of the molecular weight of PVP on the silica nanoparticles deposited onto PS surface (Fig. 8). As shown in Fig. 8a-d, with increasing molecular weight from $10 \mathrm{~kg} \mathrm{~mol}^{-1}$, $40 \mathrm{~kg} \mathrm{~mol}{ }^{-1}, 58 \mathrm{~kg} \mathrm{~mol}^{-1}$ to $130 \mathrm{~kg} \mathrm{~mol}^{-1}$, the final residue weight ratio of the composite microspheres was $12.21 \mathrm{wt} \%$, $20.82 \mathrm{wt} \%, 24.51 \mathrm{wt} \%$ and $38.71 \mathrm{wt} \%$, respectively. This result

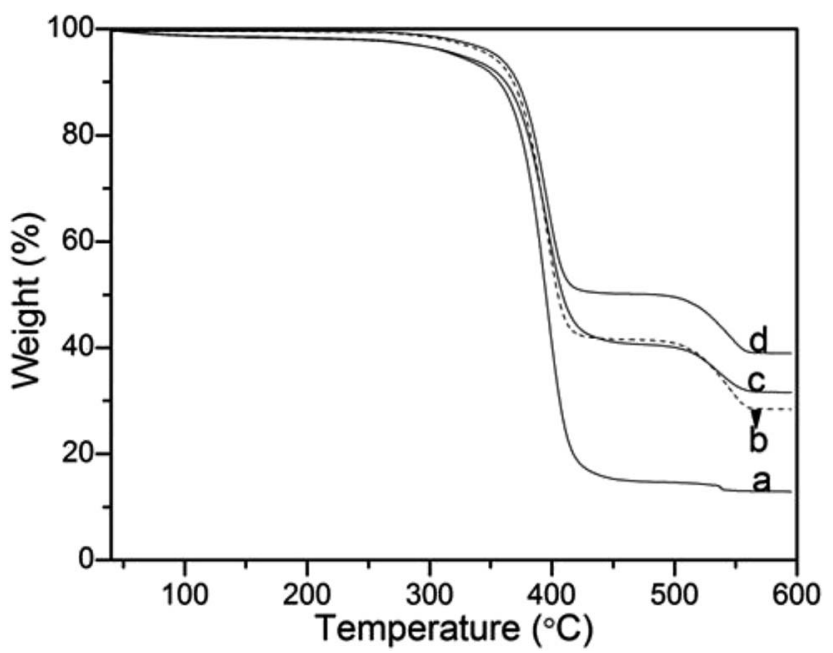

Fig. 8 Thermogravimetric analysis (TGA) curves of (a) PVP-10; (b) PVP-40, (c) PVP-58 and (d) PVP-130. $\mathrm{NH}_{3} \cdot \mathrm{H}_{2} \mathrm{O}=2 \mathrm{~mL}$. demonstrated that more silica was incorporated onto the PS microspheres, which was accordance with the analyses above.

\section{Wettability of coatings assembly from dual sized PS@SiO microspheres}

As is well known that superhydrophobic surface can be obtained by lowering the surface energy and adjusting the surface roughness. Raspberry-like composite microspheres which had uniform sizes and regular hierarchical surface roughness were the ideal building blocks for constructing superhydrophobic films. The schematic illustration of procedure for construction of superhydrophobic surfaces based on hydrophobic raspberrylike PS@SiO $\mathrm{Sim}_{2}$ composite microspheres was shown in Scheme 2.

So in this study, the influence of the surface roughness on the superhydrophobic property was investigated. The hydrophobic property can be reflected by the water contact angle (WCA) of a water droplet on the as-prepared surface. The film which was made from pure micro-sized PS microspheres showed a water CA of $95.7^{\circ}$, which was a little higher than that of smooth PS film. This was because the PS microspheres were prepared via dispersion polymerization using PVP as stabilizer, the surface of the microspheres were anchored by hydrophilic PVP that reduce the surface hydrophobicity which was also accordance with other reports. ${ }^{57}$ After hydrophobization treatment with HDTMS, the raspberry-like $\mathrm{PS@SiO}{ }_{2}$ composite microspheres were used to fabricate hydrophobic film forming a 3D multi-scales regular hierarchical surface. Accordingly, the particulate films comprised of the as-prepared raspberry-like $\mathrm{PS} @ \mathrm{SiO}_{2}$ microspheres all showed enhanced hydrophobic feature. In detail, the water CA increased from $147.2^{\circ}$ (Fig. 9c) to $153.5^{\circ}$ (Fig. 9d) and $155.1^{\circ}$ (Fig. 9e), and further to $163.3^{\circ}$ (Fig. 9f), with the corresponding roughened of the raspberrylike $\mathrm{PS}_{\mathrm{S}} \mathrm{SiO}_{2}$ microspheres. Among them, the samples showed in Fig. 9d and e exhibited superhydrophobic properties against water, on which the water droplet is in Cassie-Baxter state.

The results mentioned above indicated that the water contact angles of the particulate films were closely related to the surface roughness of the raspberry-like $\mathrm{PS@SiO}{ }_{2}$ composite microspheres. With increasing the molecular weight of the PVP used, the surface roughness of the microspheres obtained correspondingly increased (Fig. 3d and 6), which altered the geometry of their built films. According to the Wenzel and Cassie-Baxter model, magnified hydrophobicity could be approached by increasing the surface roughness. Therefore, adjusting the rough structure of the particles can effectively

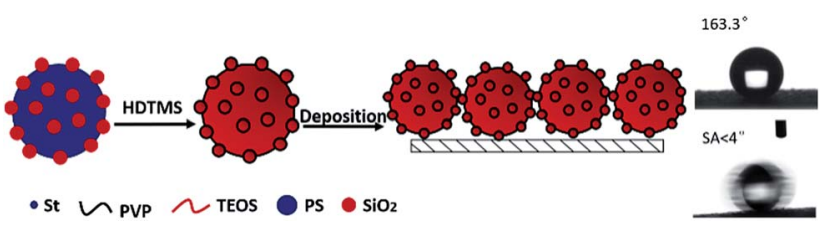

Scheme 2 Schematic illustration of procedure for construction of superhydrophobic surfaces based on hydrophobic raspberry-like $\mathrm{PS} \mathrm{aSiO}{ }_{2}$ composite microspheres. 


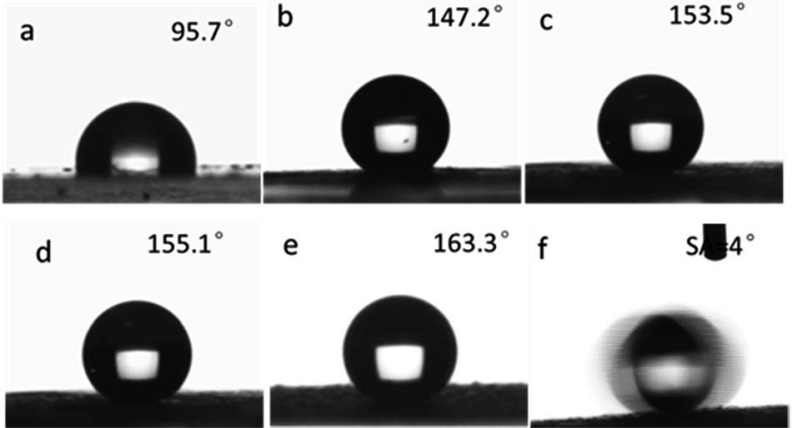

Fig. 9 Shapes of water droplets $(5 \mu \mathrm{L})$ on the films comprised of: (a) micro-sized PS; raspberry-like $\mathrm{PS} \mathrm{SSiO}_{2}$ composite microspheres prepared by using different molecular weight of PVP: (b) $10 \mathrm{~kg} \mathrm{~mol}^{-1}$; (c) $40 \mathrm{~kg} \mathrm{~mol}^{-1}$; (d) $58 \mathrm{~kg} \mathrm{~mol}^{-1}$; (e) $130 \mathrm{~kg} \mathrm{~mol}^{-1}$ and (f) captured image of the movement state of a water droplet $(5 \mu \mathrm{L})$ on the lifted ssurface. PS $\mathrm{CSiO}_{2}$ composite microspheres: $\mathrm{NH}_{3} \cdot \mathrm{H}_{2} \mathrm{O}=2.0 \mathrm{~mL}$.

tune the wettability of the film. Enhanced roughness can endow film with the ability to trap a very large fraction of air, which can repel the permeation of water as a result forming more hydrophobic surface. Moreover, the water droplet could easily slide on the lifted surface with a very low tilt angle of $4^{\circ}$, as shown in the time-lapse images in Fig. 9f and Movie S1. $\dagger$

The above mentioned method paved an avenue for further biomimic the lotus leaves surface structure. And this kind of raspberry-like particles could be used in many porous materials such as paper, cotton, sponge and steel mesh (shown in Fig. 10). These superhydrophobic/superoleophilic porous substrates can further be utilized for oil-water separation.

In this study, hydrophobicity-modified steel mesh with rigid frame was representatively selected for oil/water separation. The surface morphologies of the pristine and modified steel meshes were characterized using FE-SEM (Fig. 11a). Fig. 11a $\mathrm{a}_{1}$ showed that the pristine steel mesh had a smooth surface. After modified with raspberry-like particles, it could be seen that the particles were closely attached to the surface of the steel mesh forming the typical micro/nano multi-scale hierarchical structure (Fig. $11 \mathrm{a}_{2}$ and $\mathrm{a}_{3}$ ). Meanwhile, the pore size of the modified mesh retained almost the same, which ensures free passage of liquids through the modified mesh. Moreover, the water contact angle of the modified steel mesh was $160.6^{\circ}$, while the

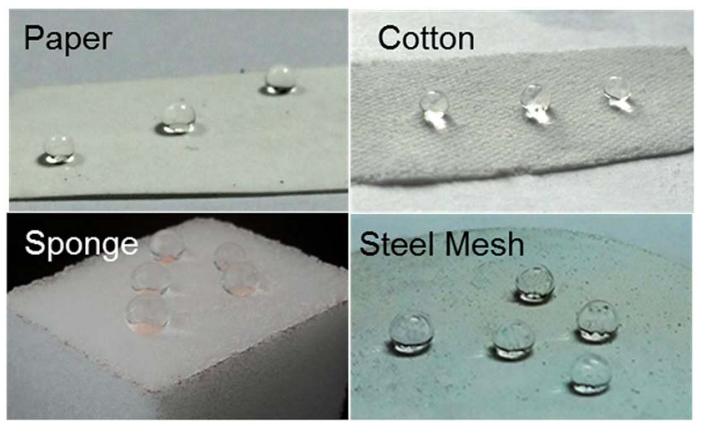

Fig. 10 Optical images of water droplets on microspheres treated (a) paper, (b) cotton, (c) sponge and (d) steel mesh.

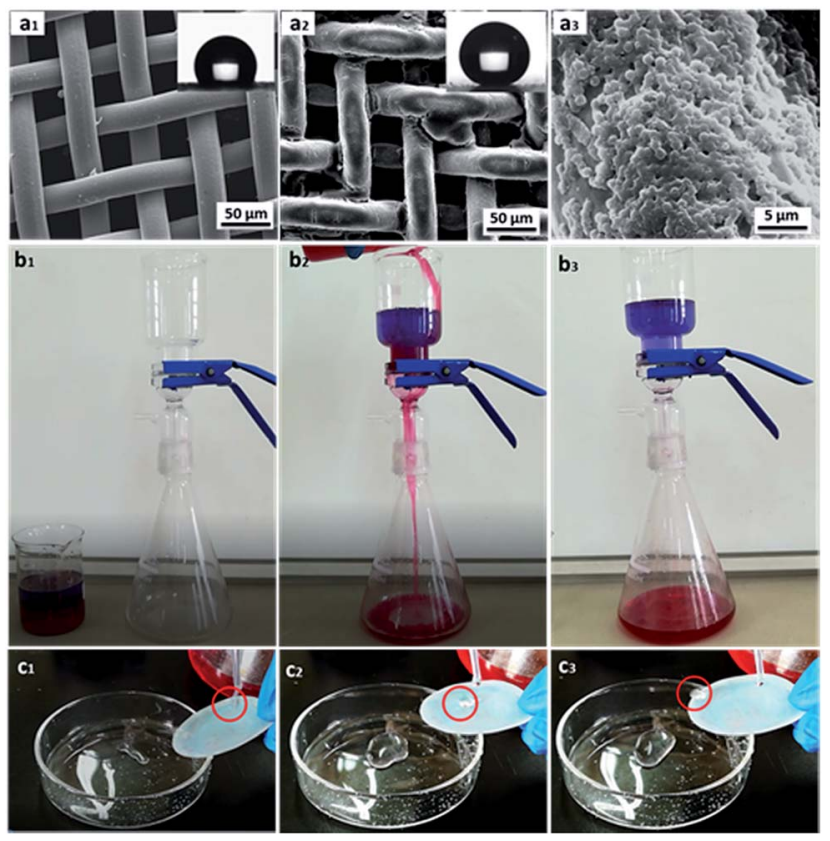

Fig. 11 (a) SEM images of pristine $\left(a_{1}\right)$, modified steel mesh $\left(a_{2}\right)$, higher magnification image of the modified steel mesh $\left(a_{3}\right)$; the inset in $\left(a_{1}\right)$ and $\left(a_{2}\right)$ were the optical image of water CA of steel mesh and modified steel mesh, respectively. (b) Sequential snapshots of the as-prepared steel mesh-based oil/water separation process of methylene blue dyed water and red oil dyed chloroform. (c) Optical pictures of superhydrophobic coated copper mesh after oil-water separation test.

water contact angle of pristine steel mesh was only $121.7^{\circ}$. This demonstrated that the modified of raspberry-like particles had endowed the steel mesh excellent superhydrophobic/ superoleophilic property. ${ }^{58}$

Then, chloroform-water mixture (200 mL, 1/1, v/v) was chosen as a representative separation test to evaluate the oilwater separation efficiency, which was shown in Fig. 11b. When the blue-dyed water was added to the funnel, it retained on the upper part of the mesh while the red-dyed chloroform quickly spread and permeated through the as prepared steel mesh and rapidly dropped into the conical flask. And there was no visible blue dyed water in the chloroform, since water was retained on the upper part of the mesh due to its excellent water repellence (see Movie S2 in the ESI $\dagger$ ). Moreover, after the oil-water separation experiment, the mesh still showed excellent superhydrophobic properties, which implied that the steel mesh modified with raspberry-like microspheres showed robust superhydrophobicity even under oil immersion and could be used recycled (see Fig. 11c and Movie S3 in the ESI $\dagger$ ).

The separation efficiency of the superhydrophobic/ superoleophilic steel mesh was also investigated. The separation efficiency was calculated by the weight ratio between the water before and after the separation process. The separation efficiency of the steel mesh was calculated up to $95.4 \%$ for the chloroform-water mixture after 5 recycle times, as shown in Fig. 12a.

As important criteria for practical applications, the recyclability of the steel mesh were also investigated. As shown in 


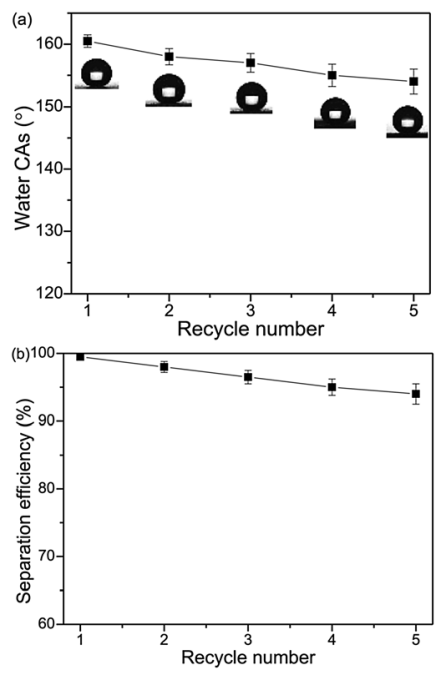

Fig. 12 Variation of the water CAs (a) and the separation efficiencies (b) of the steel mesh versus the recycle numbers by taking chloroform-water as an example.

Fig. 12b, the water contact angles of modified steel mesh decreased slightly from $160.6^{\circ}$ to $153.8^{\circ}$. The steel mesh still retained superhydrophobicity after five separation cycles.

Besides, when the oil density is smaller than that of water $\left(\rho_{\text {oil }}<\rho_{\text {water }}\right.$ ), the PU sponges can be used for the removal oil from an oil/water mixture (see Fig. S2 and Movie S4 in the ESI $\dagger$ ). These results suggested that the as-prepared porous materials had excellent superhydrophobicity and can rapidly separate oilwater mixture at large scale.

\section{Conclusions}

In conclusion, we presented a facile route for the synthesis of raspberry-like PS@SiO $\mathrm{Sim}_{2}$ composite microspheres which could further used for the fabrication of superhydrophobic surfaces. In this procedure, the PVP used are not only involving in dispersion polymerization forming monodispersed PS microspheres, but also playing a pivotal role in the biomimetic silification, since the tertiary amine sites can strongly adsorb TEOS and the biomimetic silification. The surface geometry of these raspberry-like $\mathrm{PS@SiO} \mathrm{S}_{2}$ microspheres can be well tailored by simply adjusting the molecular weight of PVP and the $\mathrm{NH}_{3} \cdot \mathrm{H}_{2} \mathrm{O}$ concentration. Furthermore, superhydrophobic surfaces can be subsequently obtained via a hydrophobization modification of these dual-scaled raspberry-like PS@SiO ${ }_{2}$ microspheres, exhibiting a water contact angle of $163.3^{\circ}$ and water contact angle hysteresis of $4^{\circ}$. Furthermore, steel mesh treated with hydrophobized raspberry-like $\mathrm{PS} @ \mathrm{SiO}_{2}$ microspheres showed excellent oil-water separation efficiency and reusability. It is believed that this facile and robust method for construction the superhydrophobic surface hold many potential application in selfcleaning and oil-water separation in large-scale.

\section{Conflicts of interest}

There are no conflicts of interest to declare.

\section{Acknowledgements}

This work was supported by the High-Level Talent Start-Up Research Project of Foshan University (gg040945).

\section{Notes and references}

1 X. Ding, S. Zhou, G. Gu and L. Wu, J. Mater. Chem., 2011, 21, 6161-6164.

2 M. G. Salvaggio, R. Passalacqua, S. Abate, S. Perathonera, G. Centib, M. Lanzac and A. Stassi, Sol. Energy, 2016, 12, 5227-5242.

3 S. Liu, S. S. Latthe, H. Yang, B. Liu and R. Xing, Ceram. Int., 2015, 41, 11719-11725.

4 Y. Lai, Y. Tang, J. Gong, D. Gong, L. Chi, C. Lin and Z. Chen, J. Mater. Chem., 2012, 22, 7420-7426.

5 Y. Wang, L. Zhang, Y. Hu and C. Li, J. Mater. Sci. Technol., 2015, 31, 901-906.

6 K. Ellinas, S. P. Pujari, D. A. Dragatogiannis, C. A. Charitidis, A. Tserepi, H. Zuilhof and E. Gogolides, ACS Appl. Mater. Interfaces, 2014, 6, 6510-6524.

7 Y. Lu, S. Sathasivam, J. Song, C. R. Crick, C. J. Carmalt and I. P. Parkin, Science, 2015, 347, 1132-1135.

8 L. Shen, B. Wang, J. Wang, J. Fu, C. Picart and J. Ji, ACS Appl. Mater. Interfaces, 2012, 4, 4476-4483.

9 Z. Wang, Y. Su, Q. Li, Y. Liu, Z. She, F. Chen, L. Li, X. Zhang and P. Zhang, Mater. Charact., 2015, 99, 200-209.

10 B. Bai, N. Quici, Z. Li and G. L. Puma, Chem. Eng. J., 2011, 170, 451-456.

11 Z. Zhou and X. Wu, Mater. Lett., 2015, 160, 423-427.

12 M. W. Lee, S. An, S. S. Latthe, C. Lee, S. Hong and S. S. Yoon, ACS Appl. Mater. Interfaces, 2013, 5, 10597-10604.

13 Q. Wang, M. Yu, G. Chen, Q. Chen and J. Tai, BioResources, 2017, 12, 643-654.

14 X. Zhou, Z. Zhang, X. Xu, F. Guo, X. Zhu, X. Men and B. Ge, ACS Appl. Mater. Interfaces, 2013, 5, 7208-7214.

15 W. Zhang, Y. Zhu, X. Liu, D. Wang, J. Li, L. Jiang and J. Jin, Angew. Chem., Int. Ed., 2014, 53, 856-860.

16 J. Li, L. Yan, X. Tang, H. Feng, D. Hu and F. Zha, Adv. Mater. Interfaces, 2016, 3, 1500770-1500777.

17 J. Li, D. Li, Y. Yang, J. Li, F. Zha and Z. Lei, Green Chem., 2016, 18, 541-549.

18 J. Li, R. Kang, X. Tang, H. She, Y. Yang and F. Zha, Nanoscale, 2016, 8, 7638-7645.

19 J. Li, C. Xu, Y. Zhang, R. Wang, F. Zha and H. She, J. Mater. Chem. A, 2016, 4, 15546-15553.

20 Y. Wang, X. Li, H. Hu, G. Liu and M. Rabnawaz, J. Mater. Chem. A, 2014, 2, 8094-8102.

21 L. Zhang, J. Wu, M. N. Hedhili, X. Yang and P. Wang, J. Mater. Chem. A, 2015, 3, 2844-2852.

22 L. Li, L. Liu, J. Lei, J. He, N. Li and F. Pan, J. Mater. Chem. A, 2016, 4, 12334-12340.

23 P. Zhang, H. Chen, L. Zhang, Y. Zhang, D. Zhang and L. Jiang, J. Mater. Chem. A, 2016, 4, 12212-12220.

24 M. Tuominen, H. Teisala, J. Haapanen, J. M. Mäkelä, M. Honkanen, M. Vippola, S. Bardage, M. E. P. Wålinder and A. Swerin, Appl. Surf. Sci., 2016, 389, 135-143. 
25 N. Wang, D. Xiong, S. Pan, Y. Deng, Y. Shi and K. Wang, Appl. Surf. Sci., 2016, 389, 354-360.

26 I. O. Arukalam, E. E. Oguzie and Y. Li, J. Colloid Interface Sci., 2016, 484, 220-228.

27 Y. Si, F. Yang and Z. Guo, J. Colloid Interface Sci., 2016, 484, 173-182.

28 W. Wang, K. Lockwood, L. M. Boyd, M. D. Davidson, S. Movafaghi, H. Vahabi, S. R. Khetani and A. K. Kota, ACS Appl. Mater. Interfaces, 2016, 8, 18664-18668.

29 Y. Lin, G. J. Ehlert, C. Bukowsky and H. A. Sodano, ACS Appl. Mater. Interfaces, 2011, 3, 2200-2203.

30 A. Davis, Y. H. Yeong, A. Steele, I. S. Bayer and E. Loth, ACS Appl. Mater. Interfaces, 2014, 6, 9272-9279.

31 U. Manna and D. M. Lynn, ACS Appl. Mater. Interfaces, 2013, 5, 7731-7736.

32 S. Pana, N. Wang, D. Xiong, Y. Deng and Y. Shi, Appl. Surf. Sci., 2016, 389, 547-553.

33 F. Li, Y. Tu, J. Hu, H. Zou, G. Liu, S. Lin, G. Yang, S. Hu, L. Miao and Y. Mo, Polym. Chem., 2015, 6, 6746-6760.

34 J. H. Yim, V. Rodriguez-Santiago, A. A. Williams, T. Gougousi, D. D. Pappas and J. K. Hirvonen, Surf. Coat. Technol., 2013, 234, 21-32.

35 R. Jafari, S. Asadollahi and M. Farzaneh, Plasma Chem. Plasma Process., 2013, 33, 177-200.

36 Y. Sun, Y. Yin, M. Chen, S. Zhou and L. Wu, Polym. Chem., 2013, 4, 3020-3027.

37 C. Zhao and A. P. J. Middelberg, RSC Adv., 2013, 3, 2122721230.

38 X. Fan, X. Jia, Y. Liu, B. Zhang, C. Li, Y. Liu, H. Zhang and Q. Zhang, Polym. Chem., 2015, 6, 703-713.

39 S. Mehlhase, C. G. Schafer, J. Morsbach, L. Schmidt, R. Klein, H. Frey and M. Gallei, RSC Adv., 2014, 4, 41348-41352.

40 F. Li, Y. Tu, J. Hu, H. Zou, G. Liu, S. Lin, G. Yang, S. Hu, L. Miao and Y. Mo, Polym. Chem., 2015, 6, 6746-6760.

41 T. Ren, J. Wang, J. Yuan, M. Pan, G. Liu, G. Zhang, G. Zhong and Z. Li, RSC Adv., 2015, 5, 36845-36857.
42 C. Du, N. Zhang, S. Ding, X. Gao, P. Guan and X. Hu, Polym. Chem., 2016, 7, 4531-4541.

43 R. Guo, X. Chen, X. Zhu, A. Dong and J. Zhang, RSC Adv., 2016, 6, 40991-41001.

44 X. Du, X. Liu, H. Chen and J. He, J. Phys. Chem. C, 2009, 113, 9063-9070.

45 Z. Qian, Z. Zhang, L. Song and H. Liu, J. Mater. Chem., 2009, 19, 1297-1304.

46 H. Zhu, Q. Zhang and S. Zhu, Dalton Trans., 2015, 44, 1675216757.

47 Q. Shang, M. Wang, H. Liu, L. Gao and G. Xiao, Polym. Compos., 2013, 34, 51-57.

48 Y. Cai, H. Xie, J. Sun, H. Liu, J. Wang, Y. Zhou, W. Nie and L. Son, Mater. Chem. Phys., 2013, 137, 796-801.

49 Z. Li, C. Wu, K. Zhao, B. Peng and Z. Deng, Colloids Surf., A, 2015, 470, 80-91.

50 D. Xu, M. Wang, X. Ge, M. H. Lamb and X. Ge, J. Mater. Chem., 2012, 22, 5784-5791.

51 X. Fan, X. Jia, Y. Liu, B. Zhang, C. Li, Y. Liu, H. Zhang and Q. Zhang, Polym. Chem., 2015, 6, 703-713.

52 F. Caruso, R. A. Caruso and H. Mohwald, Science, 1998, 282, 1111-1114.

53 Q. Shang, M. Wang, H. Liu, L. Gao and G. Xiao, Polym. Compos., 2013, 34, 51-57.

54 C. Graf, D. L. J. Vossen, A. Imhof and A. V. Blaaderen, Langmuir, 2003, 19, 6693-6700.

55 L. Jia, L. Tong, Y. Liang, A. Petretic, G. Guerin, I. Manners and M. A. Winnik, J. Am. Chem. Soc., 2014, 136, 16676-16682.

56 J. Song, F. Tronc and M. A. Minnik, J. Am. Chem. Soc., 2004, 126, 6562-6563.

57 R. Wang, H. Liu and F. Wang, Langmuir, 2013, 29, 1144011448.

58 Q. Wang, M. Yu, G. Chen, Q. Chen and J. Tian, J. Mater. Sci., 2017, 52, 2549-2559. 